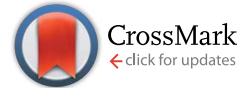

Cite this: RSC Adv., 2017, 7, 13754
Received 26th October 2016 Accepted 22nd February 2017

DOI: 10.1039/c6ra25861a

rsc.li/rsc-advances

\section{Cu nanoparticles immobilized on montmorillonite by biquaternary ammonium salts: a highly active and stable heterogeneous catalyst for cascade sequence to indole-2-carboxylic esters $\uparrow$}

\author{
Wencheng Lang, Qin Yang, Xueping Song, Mengyun Yin and Limei Zhou* \\ Copper nanoparticles immobilized on montmorillonite (MMT) by biquaternary ammonium salts $\left(N^{1}, N^{6}\right.$-dibenzyl- \\ $N^{1}, N^{1}, N^{6}, N^{6}$-tetramethylheptane-1,6-diaminium bromide, Q) were prepared by cation-exchange and \\ impregnation-reduction and designated $\mathrm{Cu}-\mathrm{Q}-\mathrm{MMT}$. The material was extensively characterized by various \\ characterization techniques such as FTIR, XRD, XPS, SEM, TEM, and $\mathrm{N}_{2}$ adsorption-desorption. The Cu-Q- \\ MMT could be used as a highly active heterogeneous catalyst for cascade sequence to indole-2-carboxylic \\ esters from ortho-bromobenzaldehydes with ethyl acetamidoacetate. Even for inactive chlorobenzaldehydes, \\ a good yield could be obtained. In addition, the catalyst can be reused six times without any significant loss of \\ activity. The high activity and stability of the Cu-Q-MMT catalyst is mainly attributed to the excellent \\ synergistic effects of biquaternary ammonium salts, Cu nanoparticles and the nanospace structure of MMT.
}

\section{Introduction}

Compared to homogeneous catalysts, heterogeneous catalysts have more advantages such as easy-separation and recyclability. ${ }^{\mathbf{1 , 2}}$ The application of heterogeneous catalysts in synthetic chemistry is attracting more attention because of their significance from both economic and ecological points of view., Montmorillonite (MMT), which is a type of naturally available clay, can be structurally defined as layers of negatively charged two-dimensional silicate sheets that are separated by interlayer cationic species with high exchange ability for other cations. ${ }^{5,6}$ Some quaternary ammoniums such as CTAB, DTAB, are widely used as surfactants to modify montmorillonites which are applied to adsorption, degradation, and polymerization and in retardants. $^{7-11}$ In addition, the organo-montmorillonites are considered as robust stabilizing materials for metal nanoparticles to form clay catalysts. ${ }^{5,12}$ For example, Manikandan and coworkers synthesized Pt-CTA-MMT for the hydrogenation of cinnamaldehyde. ${ }^{13}$ Our group reported a supported $\mathrm{Ru}$ catalyst by using CTAB intercalated montmorillonite. The catalyst exhibited high activity and selectivity in the hydrogenation of quinoline. However, it is not negligible that the stability of the catalyst is poor due to the drop of surfactants. ${ }^{7}$ Inspired by the parallel bar sport that a man with two hands holding on the bar can insist longer than the one with only one

Chemical Synthesis and Pollution Control Key Laboratory of Sichuan Province, China West Normal University, Nanchong 637002, Sichuan, China.E-mail: cwnuzhoulimei@ 163.com

$\dagger$ Electronic supplementary information (ESI) available. See DOI: 10.1039/c6ra25861a hand, we suppose that Gemini quaternary ammonium with two cation positions can anchor on the layer of montmorillonite more stably.

The indole motifs are important substructure in numerous synthetic alkaloids. ${ }^{\mathbf{1 4 - 1 8}}$ Their extensive use in the field of biology and pharmaceutics has motivated researchers to promote new synthetic strategies. ${ }^{19-22}$ Although studies on the synthesis of indole derivatives have achieved a lot, the preparation of some specific substituted patterns remains difficult such as 2-substituted-indole derivatives. ${ }^{23,24}$ As far as we all know, many indole-2-carboxylic esters and its derivatives are used as powerful precursors for antimicrobial, anticonvulsant and anticancer drugs. ${ }^{25-28}$ Usually, the synthesis of indole-2carboxylic esters contains a build of $\mathrm{C}-\mathrm{N}$ bond. Recently, $\mathrm{Cu}$ is reported as a cheap catalyst for the synthesis of indole-2carboxylic esters. In 2009, Cai group described copper salts catalyzed one-pot synthesis of indole-2-carboxylic esters from ortho-halobenzaldehyde. ${ }^{29}$ Following, several groups has reported some similar methods with copper catalyzed this kind of reaction. ${ }^{30-32}$ However, all of the reported methods in the literature to obtain the desired product are homogeneous catalysis systems which have some drawbacks: the separation of the catalyst from the reaction mixture, lack of adequate catalyst recycling methods and the residue of relatively high metal as contamination for the products. The latter problems are intolerable in the context of biological applications. To solve these problems, we immobilized $\mathrm{Cu}$ nanoparticles on montmorillonite by Gemini quaternary ammonium salts (Cu-Q-MMT) (Fig. 1) and used it as heterogeneous catalyst for cascade sequence to indole-2-carboxylic esters. 


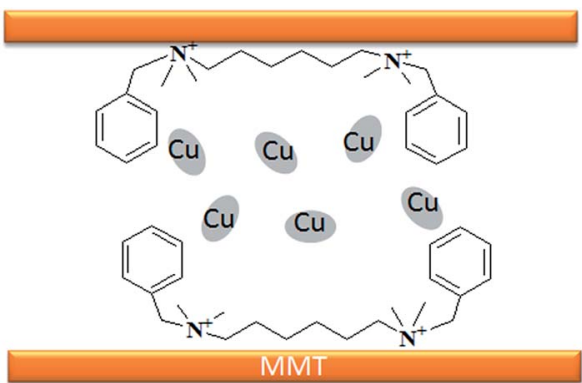

Fig. 1 A schematic illustration of Cu-Q-MMT catalyst.

\section{Experimental}

\section{Materials}

Montmorillonite powder was purchased from Alfa Aesar Company (Tianjin, China). $\mathrm{CuCl}_{2} \cdot 2 \mathrm{H}_{2} \mathrm{O}$, acetonitrile, ethanol, ethyl acetate, and $\mathrm{NaBH}_{4}$ were obtained from Kelong Chemical Reagent Co. Ltd. (Chengdu, China). All substrates for the reactions were purchased from Aladdin Industrial Inc. (America) and used without further purification. The deionized (DI) water used throughout all experiments was purified to $18.2 \mathrm{MXm}$ with the Millipore system.

\section{Synthesis of Cu-Q-MMT catalyst}

$N, N$-Dimethylbenzylamine (0.1 mol, $15.1 \mathrm{~mL}$ ), 1,6-dibromohexane $(0.025 \mathrm{~mol}, 4.0 \mathrm{~mL})$ and hydrochloric acid $(41.5 \mathrm{~mL})$ were added into a dry round bottom flask and stirred under nitrogen atmosphere. And then acetonitrile $(20 \mathrm{~mL})$ was injected as solvent. The reaction mixture was refluxed for $3 \mathrm{~h}$ before cooled down to room temperature. After rotary evaporation of the solvent, a waxy product was obtained. The waxy product repeatedly recrystallized from mixture solvent of ethanol and ethyl acetate. After dried in vacuum, the desired product was obtained as a white solid and labelled as Q. The preparation route of $\mathrm{Q}$ was shown and the structure of $\mathrm{Q}$ was confirmed by ${ }^{1} \mathrm{H}$ NMR, ${ }^{13} \mathrm{C}$ NMR and HRMS (seen in ESI, S2-5†).

Q-MMT and Cu-Q-MMT materials were prepared by a method similar to our previous reported $\operatorname{method}^{7}$ (seen in ESI, S6†).

\section{General procedure for indole-2-carboxylic esters}

Ethyl acetamidoacetate (156.2 $\mathrm{mg}, 1.2 \mathrm{mmol})$, caesium carbonate $(652 \mathrm{mg}, 2 \mathrm{mmol})$ and catalyst $(52.5 \mathrm{mg})$ were added to a dried reaction tube. The tube was flushed with nitrogen before DMSO (2 mL) was added. Then 2-bromobenzaldehyde (185 mg, $1 \mathrm{mmol}$ ) was added prior to heating the slurry to $80^{\circ} \mathrm{C}$ under a nitrogen atmosphere for $12 \mathrm{~h}$ with good agitation. After cooled down, the mixture was added DI water $(5 \mathrm{~mL})$ and extracted with ethyl acetate $(5 \mathrm{~mL})$ for 3 times. The combined organic fraction was washed with DI water $(5 \mathrm{~mL})$ and brine $(5$ $\mathrm{mL}$ ). After dried over sodium sulfate and filtered, the organic phase was concentrated to give crude product. The crude product was further purified by chromatography, eluting with dichloromethane and petroleum ether.

\section{Reuse experiment}

After completion of the cascade sequence to indole-2-carboxylic esters catalyzed by Cu-Q-MMT to afford the desired product, the catalyst was recovered by centrifugation, washed with ethyl acetate and water before dried under vacuum at $60{ }^{\circ} \mathrm{C}$ for $12 \mathrm{~h}$. The next run reaction was performed with the same amounts of reagents and solvent under the same conditions in the initial reaction.

\section{Characterization}

The FTIR spectra were recorded on an FTIR system (NICOLET 6700, Thermo Scientific Instrument). XRD measurements were performed on the D/MAX Ultima IV (Rigaku, JAPAN). X-Ray photoelectron spectroscopy (XPS) was performed using an ESCALAB $250 \mathrm{Xi}$. Transmission electron microscopy (TEM) characterization was carried out using a Tecnai G2 20 field emission electron microscope operated at $200 \mathrm{kV}$ accelerating voltage. SEM was used to determine the morphology of the catalyst system. The experiments were performed on a high-resolution scanning electron microscope (AJEOL JSM-6510LV, JAPAN). TGA was performed on a NETZSCH STA 449F3 (Piscataway, NJ) analyser in a temperature range of $25-500{ }^{\circ} \mathrm{C}$ at a heating rate of $5^{\circ} \mathrm{C} \min ^{-1}$ in $\mathrm{N}_{2}$. The $\mathrm{N}_{2}$ adsorption-desorption were calculated by utilizing a highspeed gas sorption analyzer, Autosorb-IQ (Quantachrome, U.S.A) instrument. The amount of copper in catalysts was recorded by inductively coupled plasma atomic emission spectroscopy (ICP-AES WFX-120). GC (7890 A, Agilent Technologies) with a capillary column (HP-5, $30 \mathrm{~m}$ ) was used to determine the yields of the reactions. ${ }^{1} \mathrm{H}$ NMR and ${ }^{13} \mathrm{C}$ NMR spectra were recorded on a Bruker DRX-400 instrument. HRMS spectrum was recorded on a Bruker micrOTOF-Q II instrument.

\section{Results and discussion}

\section{Characterization of $\mathrm{Cu}-\mathrm{Q}-\mathrm{MMT}$}

The FTIR spectra of MMT, Q-MMT and Cu-Q-MMT were described in Fig. 2. Compared to MMT, the spectrum of the QMMT had characteristic absorptions of native MMT, which suggested that the modification by Q did not destroy the structure of montmorillonite. Besides, the spectrum exhibited some new characterizations. The additional peaks near 2980, 2930 and $2855 \mathrm{~cm}^{-1}$ are attributed to the $\mathrm{C}-\mathrm{H}$ asymmetric and symmetric stretching vibration. ${ }^{33}$ And the peaks at 1470 and $1380 \mathrm{~cm}^{-1}$ are assigned to the bending vibration of the $\mathrm{C}-\mathrm{H}$ bonds of Q. ${ }^{34}$ The spectrum of Cu-Q-MMT is nearly the same as Q-MMT, which suggested that $\mathrm{Q}$ is successfully modified on the two materials.

The X-ray diffraction patterns supplied very useful information on the inter layer spacing of the layered material, which provides the information regarding the interlayer distance of montmorillonite. The success of the intercalation was mainly verified by measuring the increase in the basal (001) $d$-spacing. ${ }^{35}$ Fig. 3 described the X-ray diffraction patterns of MMT, Q-MMT, $\mathrm{Cu}-\mathrm{Q}-\mathrm{MMT}$ and the recycled catalyst. The curve (a) represents the XRD pattern of MMT. It can be seen that the (001) diffraction peak is at $2 \theta=7^{\circ}$. The characteristic basal spacing $d$ is 


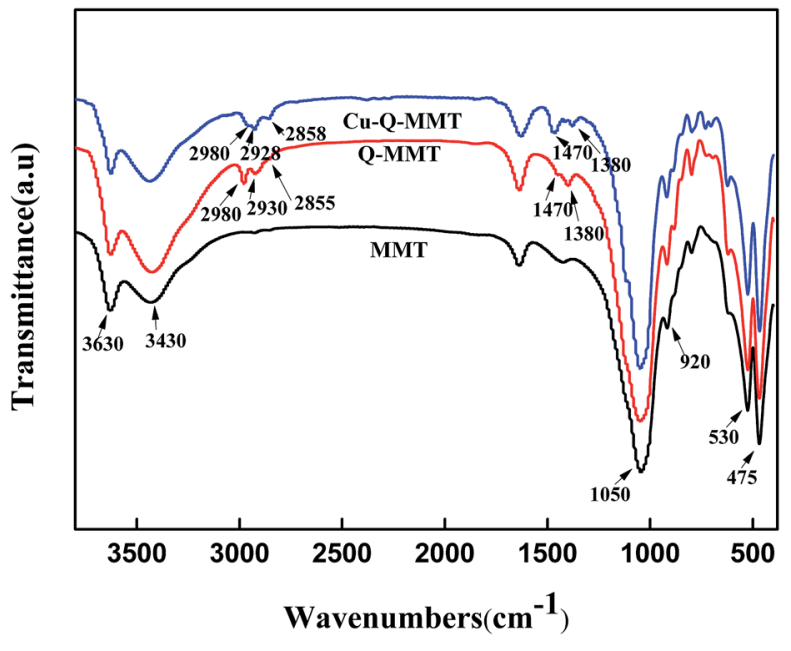

Fig. 2 FTIR analysis of MMT, Q-MMT and Cu-Q-MMT

$1.26 \mathrm{~nm}$ as calculated from Bragg's law. This reflection shifted to $5.45^{\circ}$ in the Q-MMT (curve (b)), meaning an increase in the basal spacing from $1.26 \mathrm{~nm}$ to $1.62 \mathrm{~nm}$. The enlarged $d$-spacing values indicated the intercalation of $\mathrm{Q}$ within silicate layers. When the Q-MMT was further modified with copper, this peak shifted to different angles $5.97^{\circ}$ with the basal $d$-spacing decreasing to $1.48 \mathrm{~nm}$ (curve (c)). This decrease can be explained by that parts of the unstable $\mathrm{Q}$ between the layers of montmorillonite were washed or replaced by $\mathrm{Cu}^{2+}$ in the process of impregnation-reduction. The curve (d) is the XRD pattern of the recycled catalyst. From the spectrum we cannot get its basal $d$-spacing values which indicated the (001) diffraction peak shift to the left $\left(2 \theta<3^{\circ}\right)$. The reason may be that the interlayer structure of MMT has been destroyed under basic reaction conditions.

The effective method of study the montmorillonite dispersed state in water is scanning electron microscope (SEM) and the most common method of analysis element composition is energy dispersive X-ray spectrometer (EDS). ${ }^{36}$ The SEM patterns

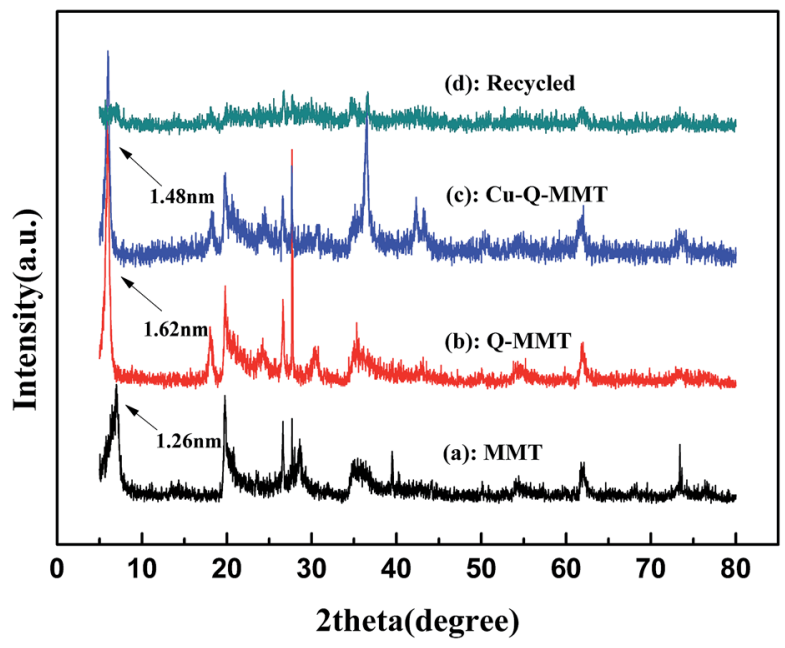

Fig. 3 XRD of MMT, Q-MMT, Cu-Q-MMT and recycled catalyst.

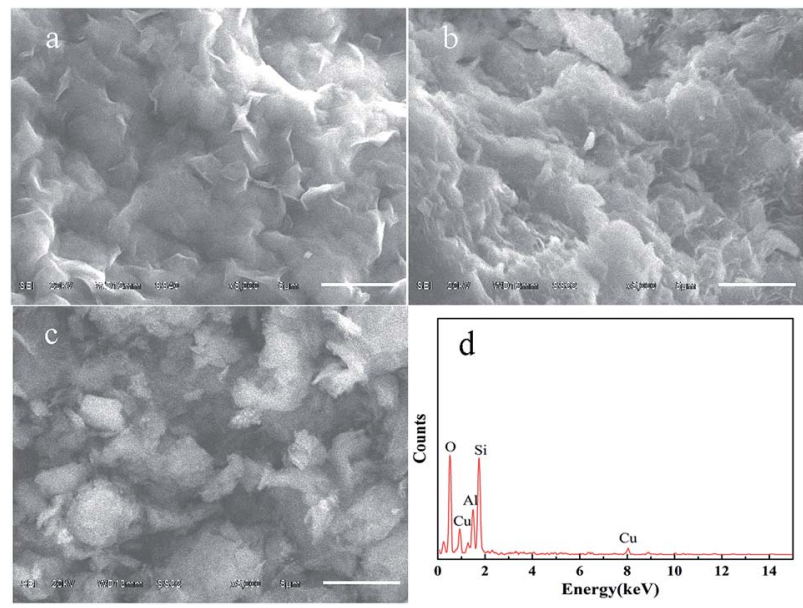

Fig. 4 SEM images of MMT, Q-MMT, Cu-Q-MMT, and EDS analysis of CU-Q-MMT.

of MMT, Q-MMT and Cu-Q-MMT are shown in the Fig. 4. The surface morphology of all samples indicated layered structure of montmorillonite. Obviously, the native MMT could disperse well in water (Fig. 4a). It is visible from the Fig. 4b that the modified Q-MMT dispersed worse in water and finally agglomerated. This phenomenon revealed that the surface of silicate platelets changed from hydrophilic one into hydrophobic one, which is caused by the interaction of alkyl chains and benzene rings. After modification of copper nanoparticles, the hydrophobic abate and agglomeration degree of $\mathrm{Cu}-\mathrm{Q}-\mathrm{MMT}$ dropped (Fig. 4c). It is confirmed by the SEM results that biquaternary ammonium salts $\mathrm{Q}$ has been successfully modified into the layers of montmorillonite. The EDS analysis also revealed the copper loaded into the montmorillonite (Fig. 4d).

The TEM micrographs of Cu-Q-MMT and the recycled catalyst are displayed in Fig. 5. As shown in Fig. 5, the morphology of copper nanoparticles is close to lamellar structure. After recycled six times, the particle size of copper nanoparticles became smaller. And a part of copper nanoparticles was lost in the recycle experiments. The amount of $\mathrm{Cu}$ loaded in virgin $\mathrm{Cu}-$ Q-MMT and recycled Cu-Q-MMT were $11.8 \%$ and $10.2 \%$, respectively.

The XPS analysis of virgin catalyst and recycled catalyst were shown in Fig. 6. It was clear that copper was in the $\mathrm{Cu}^{2+}$ and $\mathrm{Cu}^{0}$
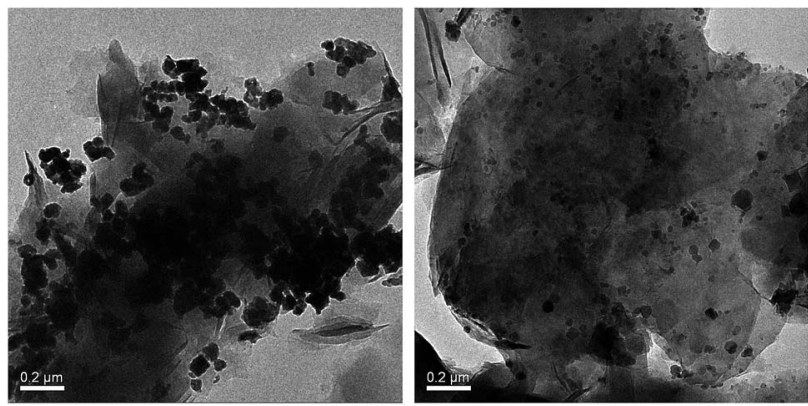

Fig. 5 TEM micrographs of virgin Cu-Q-MMT (left) and the recycled Cu-Q-MMT (right). 

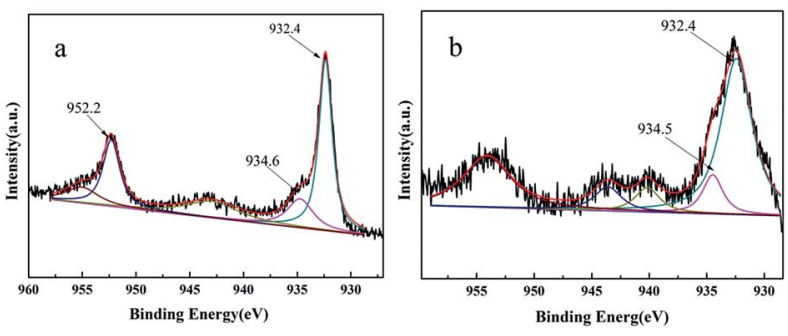

Fig. 6 XPS analysis of virgin catalyst and recycled catalyst.

components. $\mathrm{Cu} 2 \mathrm{p}_{3 / 2}$ peak at $932.4 \mathrm{eV}$ could be assigned to $\mathrm{Cu}^{0}$, whereas the peak at $934.6 \mathrm{eV}$ and $934.5 \mathrm{eV}$ could be assigned to $\mathrm{Cu}^{2+}$ (Fig. 6a and b), which probably results from oxidation of the $\mathrm{Cu}^{0}$ nanoparticles upon exposure to air. ${ }^{37}$

To study the thermal stability of the organoclay and the environments of the inserting molecules, thermogravimetric analysis was performed. The TGA curves of MMT, Q-MMT and $\mathrm{Cu}-\mathrm{Q}-\mathrm{MMT}$ were shown in Fig. 7. It is reported that the decomposition of montmorillonite occurs in two steps: desorption of water from the interlayer space occurs between 25-200 ${ }^{\circ} \mathrm{C}$ and dehydroxylation of the layer crystal lattice structure occurs between $500-800{ }^{\circ} \mathrm{C} .{ }^{38}$ The decomposition of QMMT and Cu-Q-MMT has obvious differences from MMT. Studies showed the decomposition of the organoclay often takes place in three steps: desorption of water from the interlayer space, decomposition of organic cation, and dehydroxylation of the layer crystal lattice structure. In this work the decomposition of organic cation steps occurs between 200$500{ }^{\circ} \mathrm{C}$, which also implied that the catalyst Cu-Q-MMT was relatively stable below $200{ }^{\circ} \mathrm{C}$. From all the spectra above, it can be concluded that $\mathrm{Cu}-\mathrm{Q}-\mathrm{MMT}$ has been successfully modified by $\mathrm{Q}$ and $\mathrm{Cu}$ nanoparticles.

The nitrogen adsorption-desorption isotherms of MMT, QMMT, Cu-Q-MMT and the recycle catalyst are given in Fig. 8. The isotherm on montmorillonite is of type IV characteristics according to the classification of Brunauer group, thereby indicating the mesoporous structures of all the samples. Combined with the result of XRD, it can be concluded that $\mathrm{Cu}-$ Q-MMT catalyst has nano space structure.

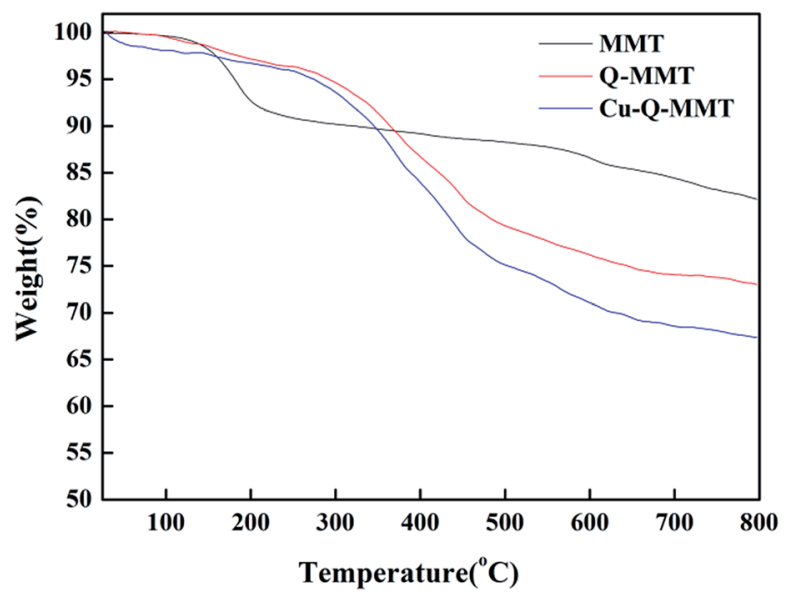

Fig. 7 TGA curves of MMT, Q-MMT and Cu-Q-MMT.
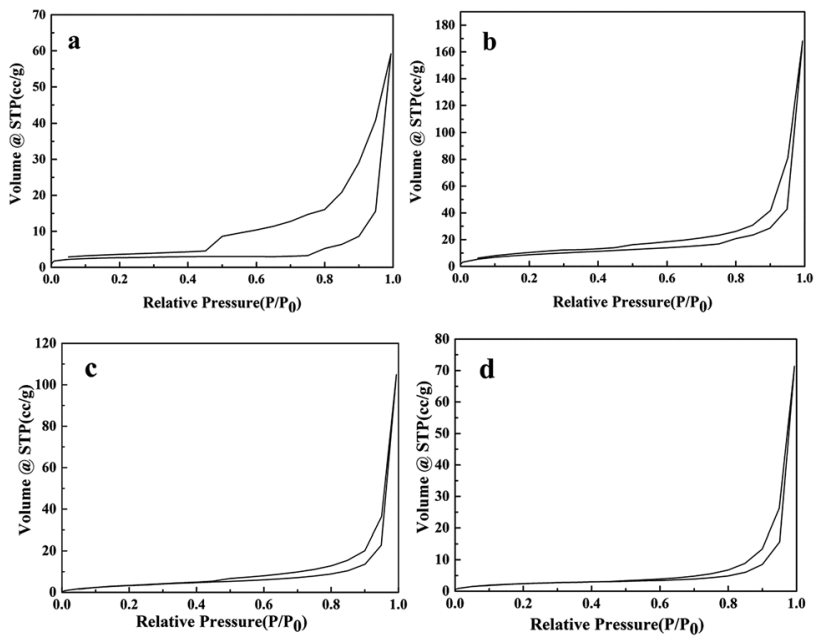

Fig. 8 The nitrogen adsorption-desorption isotherms of MMT (a), QMMT (b), Cu-Q-MMT (c) and the recycle catalyst (d).

\section{Catalysis of the cascade sequence to indole-2-carboxylic esters}

Sequentially, the catalytic performance of $\mathrm{Cu}-\mathrm{Q}-\mathrm{MMT}$ was tested in the cascade sequence to indole-2-carboxylic esters. A presumed three-step sequence that included aldol condensation, $\mathrm{C}-\mathrm{N}$ cross-coupling (Goldberg reaction), and deacylation was proposed (Scheme 1). ${ }^{30,31}$

The reaction of orthobromobenzaldehyde with ethyl acetamidoacetate was chosen as model for optimizing the reaction conditions (seen in ESI, S8-10†). And the optimal conditions were DMSO as solvent, $\mathrm{Cs}_{2} \mathrm{CO}_{3}$ as base, at $80{ }^{\circ} \mathrm{C}$, under $\mathrm{N}_{2}$ atmosphere. With the optimal conditions in hand, the catalytic performances of several catalysts were compared (Table 1).

Firstly, it can be concluded that $\mathrm{Cu}$ is important for the reaction of orthobromobenzaldehyde and ethyl acetamidoacetate to indole-2-carboxylic esters because the yield is up to $75 \%$ after the loading of $\mathrm{Cu}$ on the MMT (catalyst $\mathrm{Cu}$ MMT). $\mathrm{Cu}$ catalysts are often used in the $\mathrm{C}-\mathrm{N}$ cross-coupling reaction. ${ }^{37}$ So the $\mathrm{C}-\mathrm{N}$ cross-coupling is the key step for forming the indole-2-carboxylic esters. And with the amount of $\mathrm{Cu}$ and reaction temperature increasing, the yield of indole-2carboxylic esters increased (seen in ESI, S9 $\dagger$ ). Although the $\mathrm{Cu}-\mathrm{MMT}$ gave good yield, the activity declined obviously in the

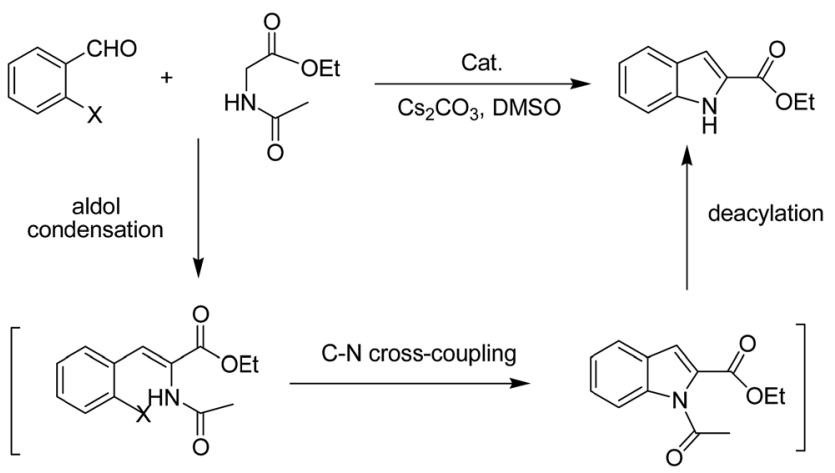

Scheme 1 Cascade sequence to indole-2-carboxylic esters. 
recycle experiments (entry 4 and 5), which also indicated its poor stability. To improve the stability of the catalyst, the MMT was modified by biquaternary ammonium salts $\mathrm{Q}$ before the loading of $\mathrm{Cu}$. Encouragingly, the catalyst Cu-Q-MMT could catalyze this reaction with a higher yield (85\%) than Cu-MMT (75\%). A possible explanation is that biquaternary ammonium salts on the MMT increase the hydrophobicity of MMT surface, benefit the adsorption of product of aldol reaction on the MMT surface, and the coordination of $\mathrm{Cu}$ on the MMT surface with product of aldol reaction, thereby enhancing the catalytic activity. Most importantly, the catalyst $\mathrm{Cu}-\mathrm{Q}-\mathrm{MMT}$ could reuse for 6 times without significant loss of activity. The results suggested that biquaternary ammonium salts $\mathrm{Q}$ modified on the MMT favor to improve the stability of catalyst as we expected. Besides, the catalyst Cu-Q-MMT showed more significant advantages: lower amount of $\mathrm{Cu}$, shorter reaction time and higher activity, comparing to the reported homogeneous catalysts CuI (entry 15 and 16). The high activity and stability of CuQ-MMT catalyst is mainly attributed to the excellent synergistic effects of biquaternary ammonium salts, $\mathrm{Cu}$ nanoparticles and the nano space structure of MMT. The nano space structure of catalyst is beneficial to improve the activity, selectivity and stability of catalyst. ${ }^{39-41}$

The generality and scope of the reaction catalyzed by $\mathrm{Cu}-\mathrm{Q}-$ MMT were also explored. The results are summarized in Table 2. And the products were characterized by ${ }^{1} \mathrm{H} \mathrm{NMR},{ }^{13} \mathrm{C} \mathrm{NMR}$ and HRMS (seen in ESI, S11-27†). Various benzaldehydes were compatible under the reaction conditions. Electronic effect and steric hindrance had a little impact on the outcome of the reaction. It is clear that the reactivity of the orthohalobenzaldehyde followed the trend of bromobenzaldehyde >

Table 1 The comparison of performance of different catalysts ${ }^{a}$

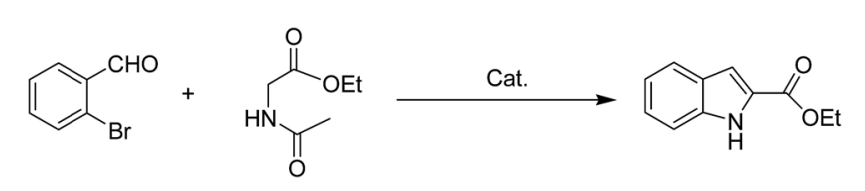

\begin{tabular}{llll}
\hline Entry & Catalyst & Conditions & Yield \\
\hline 1 & MMT & A & $18 \%$ \\
2 & Q-MMT & A & $19 \%$ \\
3 & Cu-MMT & B & $75 \%$ \\
4 & Cu-MMT(run 1) & B & $45 \%$ \\
5 & Cu-MMT(run 2) & B & $28 \%$ \\
7 & Cu-Q-MMT & B & $85 \%$ \\
8 & Cu-Q-MMT(run 1) & B & $82 \%$ \\
9 & Cu-Q-MMT(run 2) & B & $82 \%$ \\
10 & Cu-Q-MMT(run 3) & B & $82 \%$ \\
11 & Cu-Q-MMT(run 4) & B & $81 \%$ \\
12 & Cu-Q-MMT(run 5) & B & $83 \%$ \\
13 & Cu-Q-MMT(run 6) & B & $80 \%$ \\
14 & Cu-Q-MMT(run 7) & B & $72 \%$ \\
15 & CuI ${ }^{31}$ & C & $50 \%$ \\
16 & CuI & D &
\end{tabular}

${ }^{a}$ Condition A: $\mathrm{Cs}_{2} \mathrm{CO}_{3}$, DMSO, $12 \mathrm{~h}, 80{ }^{\circ} \mathrm{C}, \mathrm{N}_{2} ; \mathrm{B}: 15 \mathrm{mmol} \% \mathrm{Cu}$, $\mathrm{Cs}_{2} \mathrm{CO}_{3}$, DMSO, $12 \mathrm{~h}, 80{ }^{\circ} \mathrm{C}, \mathrm{N}_{2}$; C: $20 \mathrm{mmol} \% \mathrm{Cu}, \mathrm{Cs}_{2} \mathrm{CO}_{3}, \mathrm{DMSO}$, $16 \mathrm{~h}, 80^{\circ} \mathrm{C}$; D: $20 \mathrm{mmol} \% \mathrm{Cu}, \mathrm{Cs}_{2} \mathrm{CO}_{3}, 2-\mathrm{Me}-\mathrm{THF}, 16 \mathrm{~h}, 80^{\circ} \mathrm{C}$. chlorobenzaldehyde. So, 4-chloroindole-2-carboxylic ester was obtained as main product when 2-bromo-6-chlorobenzaldehyde was substrate (entry 7). In 2009, Cai group reported the cascade process of chlorobenzaldehyde with ethyl isocyanoacetate to indole-2-carboxylic ester at the yield of $20 \% .{ }^{29}$ Since then, there is little report on the one-pot synthesis of indole-2-carboxylic esters from chlorobenzaldehyde because of its low reactivity. In our experiment, catalyzed by Cu-Q-MMT, 2-chlorobenzaldehydes and 2,4-dichlorobenzaldehyde coupled with

Table 2 Synthesis of various indole-2-carboxylic esters ${ }^{a}$

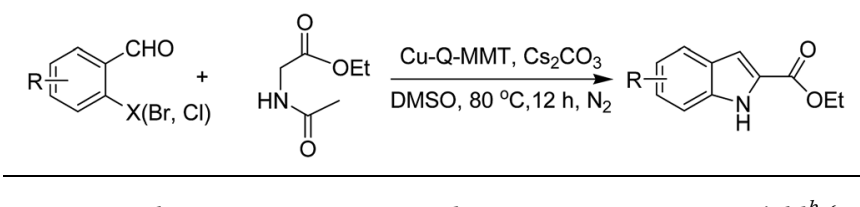

\begin{tabular}{|c|c|c|c|}
\hline Entry & Substrate & Product & Yield $^{b}(\%)$ \\
\hline
\end{tabular}

1

2

3

4<smiles>O=Cc1ccccc1Br</smiles><smiles>O=Cc1cc(F)ccc1Br</smiles><smiles>O=Cc1cc(Cl)ccc1Br</smiles><smiles>COc1ccc(Br)c(C=O)c1</smiles><smiles>O=Cc1cc(C(F)(F)F)ccc1Br</smiles><smiles>O=Cc1ccc2ccccc2c1Br</smiles>

7

(1)

8

8

$\%$

$83 \%$

$72 \%$

$5 \%$ $\%$

\footnotetext{
${ }^{a}$ Reaction conditions: $1.0 \mathrm{mmol}$ aryldehyde, $1.2 \mathrm{mmol}$ ethyl acetamidoacetate, $2.0 \mathrm{mmol} \mathrm{Cs}_{2} \mathrm{CO}_{3}$, $52.5 \mathrm{mg}$ Cu-Q-MMT, $2.0 \mathrm{~mL}$ DMSO. ${ }^{b}$ Yield was obtained after column chromatography.
} 
ethyl acetamidoacetate to the aimed indole-2-carboxylic esters gave encouraging separation yields of $68 \%$ and $75 \%$, respectively (entry 8 and 9).

\section{Conclusion}

In conclusion, the MMT supported catalyst Cu-Q-MMT was prepared by using cation-exchanged and impregnation-reduction methods. The obtained catalyst showed high activity for cascade sequence to indole-2-carboxylic esters. Remarkably, this catalyst could catalyze ethyl acetamidoacetate with inactive chlorobenzaldehydes and gave a good yield. In addition, the catalyst could be easily isolated from the reaction mixture by centrifugation and reused up to 6 times. The high activity and stability of Cu-Q-MMT catalyst is mainly ascribed to the excellent synergistic effects of biquaternary ammonium salts, $\mathrm{Cu}$ nanoparticles and the nano space structure of MMT. We believe that other transition metals could also be supported on MMT using this method and the resulting materials could be used as efficient and stable nanocatalyst.

\section{Acknowledgements}

The authors are grateful for financial support from the National Natural Science Foundation of China (21303139).

\section{References}

1 Z. Dong and Z. Ye, Appl. Catal., A, 2015, 489, 61.

2 P. G. Najrul Hussain, P. Khared and M. R. Das, RSC Adv., 2015, 5, 103105.

3 S. Sayyahi, Asian J. Chem., 2013, 25, 6471.

4 P. T. Anastas, M. M. Kirchhoff and T. C. Williamson, Appl. Catal., A, 2001, 221, 3.

5 T. Li, J. Zhang, X. Xie, X. Yin and X. An, Fuel, 2015, 143, 55. 6 S. Miao, Z. Liu, B. Han, J. Huang, Z. Sun, J. Zhang and T. Jiang, Angew. Chem., 2005, 45, 266.

7 L. Zhou, X. Qi, X. Jiang, Y. Zhou, H. Fu and H. Chen, J. Colloid Interface Sci., 2013, 392, 201.

8 S.-D. Lee, M.-S. Park, D.-W. Kim, I. Kim and D.-W. Park, Catal. Today, 2014, 232, 127.

9 H. Fan, L. Zhou, X. Jiang, Q. Huang and W. Lang, Appl. Clay Sci., 2014, 95, 150.

10 M. Kozak and L. Domka, J. Phys. Chem. Solids, 2004, 65, 441.

11 S. Miao, Z. Liu, B. Han, H. Yang, Z. Miao and Z. Sun, J. Colloid Interface Sci., 2006, 301, 116.

12 Z. Huang, P. Wu, H. Li, W. Li, Y. Zhu and N. Zhu, RSC Adv., 2014, 4, 6500.

13 D. Manikandan, D. Divakar, A. V. Rupa, S. Revathi, M. E. L. Preethi and T. Sivakumar, Appl. Clay Sci., 2007, 37, 193.
14 S. Cacchi and G. Fabrizi, Chem. Rev., 2011, 111, PR215. 15 S. Cacchi and a. G. Fabrizi, Chem. Rev., 2005, 105, 2873. 16 M. Inman and C. J. Moody, Chem. Sci., 2013, 4, 29.

17 X. Zhang, R. Guo and X. Zhao, Org. Chem. Front., 2015, 2, 1334.

18 D. F. Taber and P. K. Tirunahari, Tetrahedron, 2011, 67, 7195.

19 A. Ganesan, J. Kothandapani, J. B. Nanubolu and S. S. Ganesan, RSC Adv., 2015, 5, 28597.

20 S. Bahn, S. Imm, K. Mevius, L. Neubert, A. Tillack, J. M. Williams and M. Beller, Chemistry, 2010, 16, 3590.

21 G. Palmisano, A. Penoni, M. Sisti, F. Tibiletti, S. Tollari and K. M. Nicholas, Curr. Org. Chem., 2010, 14, 2409.

22 R. G. Vaswani, B. K. Albrecht, J. E. Audia, A. Cote, L. A. Dakin, M. Duplessis, V. S. Gehling, J. C. Harmange, M. C. Hewitt, Y. Leblanc, C. G. Nasveschuk and A. M. Taylor, Org. Lett., 2014, 16, 4114.

23 H. J. A. Sudhakara, K. M. Mahadevan and V. Hulikal, Synth. Commun., 2009, 39, 2506.

24 K. K. James McNulty, Eur. J. Org. Chem., 2011, 6902.

25 W. Sun, L. Hong and R. Wang, Chemistry, 2011, 17, 6030.

26 R. Mali and V. Yadav, Synthesis, 1984, 1984, 862.

27 S. Basceken, S. Kaya and M. Balci, J. Org. Chem., 2015, 80, 12552.

28 N. K. Kaushik, N. Kaushik, P. Attri, N. Kumar, C. H. Kim, A. K. Verma and E. H. Choi, Molecules, 2013, 18, 6620.

29 Q. Cai, Z. Li, J. Wei, C. Ha, D. Pei and K. Ding, Chem. Commun., 2009, 7581.

30 S. G. Koenig, J. W. Dankwardt, Y. Liu, H. Zhao and S. P. Singh, ACS Sustainable Chem. Eng., 2014, 2, 1359.

31 S. G. Koenig, J. W. Dankwardt, Y. Liu, H. Zhao and S. P. Singh, Tetrahedron Lett., 2010, 51, 6549.

32 Z. Zhu, J. Yuan, Y. Zhou, Y. Qin, J. Xu and Y. Peng, Eur. J. Org. Chem., 2014, 511.

33 C. Zhao, Z. Sun, B. Liu and G. Peng, J. Macromol. Sci., Part B: Phys., 2013, 52, 1453.

34 T. H. Kim, S. T. Lim, C. H. Lee, H. J. Choi and M. S. Jhon, J. Appl. Polym. Sci., 2003, 87, 2106.

35 X. Shi and Z. Gan, Eur. Polym. J., 2007, 43, 4852.

36 M. Kozak and L. Domka, J. Phys. Chem. Solids, 2004, 65, 441.

37 Q. Huang, L. Zhou, X. Jiang, Y. Zhou, H. Fan and W. Lang, ACS Appl. Mater. Interfaces, 2014, 6, 13502.

38 R. R. Tiwari, K. C. Khilar and U. Natarajan, Appl. Clay Sci., 2008, 38, 203.

39 G. Lan, Y. Yao, X. Zhang, M. Guo, H. Tang, Y. Li and Q. Yang, Catal. Sci. Technol., 2016, 6, 2181.

40 M. Zhong, Y. Zhao, Q. Yang and C. Li, J. Catal., 2016, 338, 184.

41 Y. Yao, X. Zhang, J. Peng and Q. Yang, Chem. Commun., 2015, 51, 3750. 